Aline Trabut, Égalité, Bourrache, Aristide et les autres... Les prénoms révolutionnaires dans le département des Côtes-du-Nord\&bnsp;-1789-1800 ; Alain Croix, Pascale Scilbo (dir.), Brutus, Désir de la paix, Jonquille... Les prénoms de la Révolution en Loire-Inférieure

\title{
Serge Bianchi
}

\section{(2) OpenEdition}

Édition électronique

URL : https://journals.openedition.org/ahrf/996

DOI : 10.4000/ahrf.996

ISSN : 1952-403X

Éditeur :

Armand Colin, Société des études robespierristes

Édition imprimée

Date de publication : 1 décembre 2000

Pagination : 153-155

ISSN : 0003-4436

Référence électronique

Serge Bianchi, «Aline Trabut, Égalité, Bourrache, Aristide et les autres... Les prénoms révolutionnaires dans le département des Côtes-du-Nord\&bnsp;- 1789-1800; Alain Croix, Pascale Scilbo (dir.), Brutus, Désir de la paix, Jonquille... Les prénoms de la Révolution en Loire-Inférieure ", Annales historiques de la Révolution française [En ligne], 322 | octobre-décembre 2000, mis en ligne le 26 avril 2006, consulté le 23 avril 2022. URL : http://journals.openedition.org/ahrf/996 ; DOI : https://doi.org/10.4000/ahrf.996

Ce document a été généré automatiquement le 23 avril 2022

Tous droits réservés 


\section{Aline Trabut, Égalité, Bourrache, Aristide et les autres... Les prénoms révolutionnaires dans le département des Côtes-du-Nord\&bnsp;- 1789-1800 ; Alain Croix, Pascale Scilbo (dir.), Brutus, Désir de la paix, Jonquille... Les prénoms de la Révolution en Loire- Inférieure}

Serge Bianchi

\section{RÉFÉRENCE}

Aline Trabut, Égalité, Bourrache, Aristide et les autres... Les prénoms révolutionnaires dans le département des Côtes-du-Nord - 1789-1800, Saint-Brieuc, Vive 89 en Côtes-du-Nord, 1993, 263 p. // Alain Croix, Pascale Scilbo, (dir.) Brutus, Désir de la paix, Jonquille... Les prénoms de la Révolution en Loire-Inférieure, Nantes, Nantes-Histoire, 1993, 143 p.

Dans les suites logiques des recherches initiées par le bicentenaire de la Révolution française ont été publiés la même année un ouvrage collectif sur les prénoms révolutionnaires de la Loire-Atlantique (autrefois "inférieure ») et une maîtrise, soutenue en 1991 à Rennes 2, sur le même phénomène dans les Côtes-du-Nord (aujourd'hui « d'Armor »). Une forte unité logique et conceptuelle de ces ouvrages tient à la personnalité de leur initiateur, Alain Croix, qui tente d'affiner, pour l'espace breton, des outils et méthodes expérimentés pour le Val-de-Marne. En regroupant ces 
travaux (et des maitrises analogues pour le Morbihan et l'Ille-et-Vilaine) il est possible de s'interroger sur la pertinence d'un modèle «breton" en matière de prénoms révolutionnaires ou « de la Révolution ».

2 Aline Trabut adopte un plan «classique» pour les Côtes-du-Nord, en étudiant successivement les sources et les choix, le traitement d'un corpus dans l'espace et le temps, l'interprétation sociologique et culturelle des prénoms révolutionnaires, soigneusement présentés et classés (on soulignera la qualité des annexes). Les historiens de Nantes-Histoire ont choisi de présenter leur enquête dans un article de fond volumineux, auquel succèdent des tableaux précis et un glossaire des prénoms étudiés. Si l'ampleur des ouvrages et leurs démarches diffèrent, les analogies l'emportent largement.

3 Tous deux reposent sur un ensemble de préalables communs, dont l'utilisation d'un échantillon de communes, d'un sondage établi en fonction de l'état des sources et des caractères des communes étudiées. Tandis qu'Aline Trabut s'intéresse à $15 \%$ des communes du département (67 sur 321 paroisses), les historiens nantais ont dépouillé les registres de 47 communes (et 62 registres de l'an II), soit près de la moitié de l'ensemble, et ce sur les dix années de la Révolution. Il s'agit de respecter un certain équilibre entre villes et campagnes, entre zones «bleues » et «blanches », pour tenter de mesurer les niveaux de rupture (politique culturelle), des Lumières à la Révolution. Les auteurs se refusent à toute polémique sur la qualification préalable du phénomène et proposent, à l'issue d'enquêtes de terrain, une moisson stimulante de résultats quantitatifs et qualitatifs.

4 Le bilan des quelque 104 communes étudiées est à la fois modeste et éloquent. Moins de $2 \%$ des enfants des Côtes-du-Nord (en l'an II, soit 63 prénoms de rupture) et 112 au total pour Aline Trabut. $30 \%$ des garçons et $20 \%$ des filles à Nantes (342prénoms de rupture en l'an II) mais 122 hors de la grande ville, justifiant le titre de "Nantes et le désert breton ", en matière de comportement des communes rurales. Dans un cas comme dans l'autre, la quasi-totalité des villages n'est pas "effleurée » par la pratique, comme autant de fronts de résistance à l'acculturation républicaine. Le phénomène se développe proportionnellement à la taille de la ville, avec bien des exceptions comme Tréguier dans les Côtes-du-Nord. Les statistiques s'imposent, par les distinctions apportées dans le sexe de l'enfant, son statut (illégitime ou abandonné), la chronologie de l'acte (3 avril 1791 un François Pierre National), la place des unités prénominales.

Des hypothèses se renforcent au fil des deux lectures. Les garçons sont plus nombreux à porter des prénoms «militants» (héros, valeurs de la Révolution) alors que les filles sont distinguées par des prénoms de rupture «culturelle». La pratique urbaine, importante pour les enfants illégitimes, apparaît pourtant largement spontanée. L'accent est mis sur les facteurs et les vecteurs de diffusion de tels prénoms. Les auteurs interrogent la sociologie des parents, leur engagement éventuel dans les armées, les sociétés populaires. Les prénoms révolutionnaires nantais émanent de la «sansculotterie » (artisans, ouvriers), tandis que le monde de la mer et des rivières se signale hors de l'agglomération. Par contre s'impose l'absence presque totale des agriculteurs et du monde de la terre. Les médiations de tel représentant en mission (Le Carpentier) ou de tel instituteur républicain (ou l'aubergiste) permettent parfois de faire le lien entre la vie quotidienne et la culture politique. Aline Trabut tente, de son côté, de relier la pratique des prénoms aux influences culturelles proches, telles les fêtes civiques, les pièces de théâtre, les chansons, et une "ébauche de révolution culturelle», qui 
relèverait du mythe pour Alain Croix. L'intérêt de la démarche est d'individualiser l'acte en pesant, en fonction des variables, son degré de rupture et son « ambiance ».

6 L'analyse des corpus de prénoms serait plus banale. Tandis que les valeurs de la Révolution (40\% du total, à l'exemple de Vérité) l'emportent dans les Côtes-du-Nord sur les prénoms antiques (27\%), on compte 151 Brutus en Loire-Inférieure, mais 680Rose Parmi d'autres apports des deux ouvrages l'on peut souligner la prise en compte des changements de prénoms d'adultes, ou la restitution des carrières de personnes impliquées dans la pratique des prénoms révolutionnaires. Quand la rigueur de la recherche permet de suivre la gestation du prénom révolutionnaire et de comprendre si Rose est un prénom traditionnel (porté par la marraine-témoin) ou un prénom révolutionnaire (par la fréquence ou la situation du père), on conçoit l'intérêt de ces orientations de recherche.

7 Quelques critiques peuvent nuancer ces éloges, sans altérer la qualité de l'ensemble. L'étude des champs culturels des ruptures nous a paru parfois quelque peu plaquée, voire artificielle, dans la fragilité des liens proposés avec les prénoms. Il est possible de contester le principe du sondage et la représentativité des échantillons choisis. Des erreurs d'échelle sont toujours possibles et de nombreuses communes restent en dehors de l'espace couvert par la recherche. L'accent nous parait parfois mis sur les villes plus que sur les campagnes des Côtes-du-Nord. Une enquête départementale exhaustive nous paraît plus fiable, même si les auteurs reconnaissent les lacunes et les risques de leurs choix. Les rubriques sélectionnées apportent des regroupements intéressants, sans périmer à nos yeux les grilles d'études précédentes. Peut-on séparer les valeurs de la Révolution du calendrier républicain La question est posée. Mais si l'on peut discuter la prise en compte ou le refus des Victoire et Hyacinthe en tant que prénoms révolutionnaires, le lecteur dispose toujours des moyens et de la liberté de reprendre le dossier, selon ses propres critères. Le doute n'est pas permis toutefois quand un instituteur de Plancoët prénomme son enfant Julien Vengeur AlainVictorieux, le 15 juin 1794

8 Au terme de ces lectures il est désormais possible d'esquisser un "modèle » breton d'attribution des prénoms révolutionnaires, très différents d'autres modèles connus à ce jour (Val-de-Marne et Essonne actuels, Oise...). On pourrait ajouter à ces deux ouvrages des maitrises comparables (par la méthode et les orientations de recherches) sur l'ille-et-Vilaine et le Morbihan, des articles sur le Finistère, et de nombreux travaux de maitrise, dans un cadre chronologique plus large, pour inscrire les prénoms révolutionnaires dans la moyenne durée, les évolutions d'allongement, de transmission, de choix différenciés des unités prénominales. La synthèse est dès lors tout à fait envisageable. Si la "région Bretagne » apparaît dans son ensemble (et ses campagnes) réfractaire à la rupture qu'impliquaient les prénoms révolutionnaires, des noyaux de républicains convaincus ont tenté, par cette pratique militante, de peser sur la "régénération » de leur milieu et de leur pays. C'est cette tentative minoritaire, incomprise et souvent marginalisée, que ces deux ouvrages restituent avec scrupules et rigueur. 